\title{
Las capacidades socioafectivas y el comportamiento proambiental
}

\author{
socioafective capabilities and pro environmental behavior
}

July Jacinta Solís Mendoza ${ }^{1}$

\begin{abstract}
RESUMEN
Objetivo: Determinar la relación entre el desarrollo de capacidades socioafectivas y el comportamiento pro ambiental de los niños de la Institución Educativa Inicial Nº68 del distrito de El Agustino en el año 2019. Material y método: En la investigación se utilizaron fichas de información y una encuesta; estas fueron aplicadas a 90 estudiantes de educación 4 y 5 años de la institución Educativa Inicial N 068 del Distrito El Agustino, Lima. Los instrumentos de recolección de datos fueron validados a través del juicio de expertos y su confiabilidad fue establecida mediante el coeficiente de Alfa de Cronbach con valores de 0,976 y 0,972 para el caso de la ficha de información y la encuesta respectivamente. En este trabajo de investigación se utilizó una metodología cuantitativa y un diseño descriptivo correlacional. Resultado: Se determinó que las variables de estudio tienen una relación; lineal, altamente significativa con coeficiente Rho de Spearman de 0,976. Conclusión: Existe una relación el desarrollo de las capacidades socioafectivas con el comportamiento proambiental en los niños de 4 y 5 años.
\end{abstract}

Palabras clave: socio afectividad, medio ambiente, capacidades interpersonales e intrapersonales, educación inicial, desarrollo sostenible.

\section{ABSTRACT}

Objective: To determine the relationship between the development of socio-affective capacities and the pro-environmental behavior of the children of the Initial Educational Institution No. 068 of the district of El Agustino in 2019. Material and method: In the research, information sheets and a survey; These were applied to 90 students of education 4 and 5 years of the Initial Educational Institution No. 068 of the El Agustino District, Lima. The data collection instruments were validated through expert judgment and their reliability was established using Cronbach's Alpha coefficient with values of 0,976 and 0,972 for the case of the information sheet and the survey, respectively. In this research work a quantitative methodology and a correlational descriptive design were used. Result: It was determined that the study variables have a relationship; linear, highly significant with Spearman's Rho coefficient of 0,976 . Conclusion: There is a relationship between the development of socio-affective capacities with pro-environmental behavior in children aged 4 and 5 years.

Keywords: socio-affectivity, environment, interpersonal and intrapersonal skills, initial education, sustainable development.

\section{INTRODUCCIÓN}

Nuestra actualidad muestra que hemos descuidado el medio ambiente y, por obvia razón, hemos descuidado una pieza vital para el desarrollo sustentable del mundo. En este sentido, las actuales condiciones medioambientales exigen la búsqueda de soluciones a los problemas que la sociedad ha generado en el medio ambiente. La necesidad de responder desde la educación a las demandas de una sociedad en permanente cambio y más sensibilizada con las repercusiones del hombre hacia el medio ambiente hace que, estudiemos cada vez más los diferentes sustentos pedagógicos y psicológicos para desarrollar desde niños capacidades que le permitan modificar sus actitudes y comportamientos inteligentes para contribuir con el cuidado del medio ambiente. Estudios orientados a analizar hasta qué punto las emociones o reacciones generadas ante determinadas situaciones se relacionan con comportamientos Pro Ambientales. Actualmente se debate la introducción de Educación Ambiental en las prácticas pedagógicas de los docentes, desprendiéndose propuestas, reflexiones y críticas sobre diversos temas y realidades como, las características de los soportes pedagógicos pertinentes que respondan a la formación del nivel inicial; las capacitaciones docentes para mediar en el desarrollo las capacidades socioafectivas de los niños para una consolidada y contextualizada educación ambiental que logre comportamientos Pro Ambientales, con relación a acciones orientadas al cuidado del medio ambiente, al cuidado de la salud y a la prevención de riesgos de desastres. En este contexto, la escuela debe responder a los retos que plantean el desarrollo de las capacidades socioafectivas orientadas a una Educación Ambiental que desarrolle comportamientos Pro Ambientales en los niños del nivel educativo inicial.

En relación al desarrollo de capacidades socioafectivas; Kollmus y Agyeman (2002) mencionan que el ser humano presenta emociones desfavorables que son ocasionados por el deterioro del medio ambiente. Aquello genera en él una conducta pro ambiental. Por su parte Duránd, Alzate, López y Saducedo (2007) consideran que el medio ambiente es un tema sonante hoy en día. La preocupación se ha acrecentado a tal 
punto que los ciudadanos se han dado cuenta que les afecta de manera sustancial su calidad de existencia. Papalia, Wendkos y Feldman (2007) indican por su parte que un individuo a la edad de los tres a los siete años es relevante para el desarrollo social y emocional de un infante. A partir de ese rango de edades, se irá desarrollando el yo, en entendimiento de sus emociones y se incrementarán sus acciones hacia el bienestar de su entorno.

En lo que respecta al comportamiento pro ambiental, Martínez (2004) indica que es una temática estudiada por la psicología ambiental, pues está vinculada a un comportamiento que tiene que ver con el cuidado del medio ambiente. Por su parte, Zapata y Castrechini (2011) mencionan que el comportamiento medio ambiental: "es un conjunto de actividades humanas cuya intencionalidad es contribuir a la protección de los recursos naturales, o al menos, a la reducción del deterioro ambiental; son acciones dirigidas y efectivas que responden a requerimientos sociales e individuales y que resultan en la protección del medio ambiente. Peña (2017) manifiesta que está vinculado a favor del medio ambiente; lo considera como: "un conjunto de acciones deliberadas y efectivas que responden a requerimientos sociales e individuales y que resultan en la protección del medio." (pág. 125). Hablar del comportamiento proambiental, acota la autora, tiene que ver con la disminución del deterioro y a una mejora de las condiciones actuales del contexto en el que vivimos.

Por lo mencionado, se tuvo como objetivo fundamental determinar la relación entre el desarrollo de las capacidades socioafectivas y el comportamiento Pro Ambiental de los niños de la Institución Educativa Inicial $N^{\circ} 068$ del distrito de El Agustino en el año 2019.

\section{MATERIALES Y MÉTODOS}

La investigación se realizó con un diseño descriptivo correlacional tipo cuantitativo, puesto que se buscó describir las variables; Capacidades Socioafectivas y el Comportamiento Pro Ambiental; así también se determinó encontrar la relación entre las variables.

La población, estuvo conformada por 90 de niños de 4 y 5 años de edad de la Institución Educativa Inicial $N^{\circ} 068$ del distrito de El Agustino en el año 2019 y la unidad de análisis constó de 73 niños. La selección de muestra se hizo por medio estratos tomando en cuenta la variable interviniente edad; se muestra a continuación la tabla que permite establecer dicha estratificación (Tabla 1).
Tabla 1.

Muestra estratificada proporcional de los niños de inicial

\begin{tabular}{cccc}
\hline $\begin{array}{c}\text { Estratos de } \\
\text { la } \\
\text { población } \\
\text { de niños. }\end{array}$ & $\begin{array}{c}\text { Tamaño } \\
\text { de la } \\
\text { población } \\
\text { de cada } \\
\text { estrato. }\end{array}$ & $\begin{array}{c}\text { Factor de } \\
\text { proporcional } \\
\text { idad* (f) }\end{array}$ & $\begin{array}{c}\text { Estratos } \\
\text { de la } \\
\text { muestra. }\end{array}$ \\
\hline $\begin{array}{c}\text { Niños de } 4 \\
\text { años }\end{array}$ & 28 & 0,81 & 23 \\
$\begin{array}{c}\text { Niños de } 5 \\
\text { años }\end{array}$ & 62 & 0,81 & 50 \\
\hline Total & 90 & Total & 73 \\
\hline
\end{tabular}

Tabla 2.

Técnicas, instrumentos e informantes

\begin{tabular}{lll}
\hline Técnicas & Instrumentos & Informantes \\
\hline $\begin{array}{l}\text { Observación } \\
\text { Encuestas }\end{array}$ & $\begin{array}{l}\text { Ficha de observación } \\
\text { Cuestionarios }\end{array}$ & $\begin{array}{l}\text { Niños } \\
\text { Docentes }\end{array}$ \\
\hline
\end{tabular}

Los instrumentos de recojo de información que fueron aplicados por y a los docentes, como parte de los sujetos informantes de la muestra de estudio: Ficha de observación aplicado por docentes de Inicial a los niños de la muestra $(n=73)$. Cuestionario aplicado a docentes de la muestra $(n=10)$. 
Tabla 3.

Criterios técnicos para el procesamiento de datos de la Ficha de Observación

\begin{tabular}{|c|c|c|c|c|c|c|}
\hline VARIABLE & DIMENSIONES & $\begin{array}{l}\mathrm{N}^{\circ} \text { de } \\
\text { Ítem }\end{array}$ & $\begin{array}{c}\text { Cant. de } \\
\text { Ítems }\end{array}$ & $\begin{array}{l}\text { Escala por } \\
\text { indicadores }\end{array}$ & $\begin{array}{c}\text { Rangos } \\
X \\
\text { dimensiones }\end{array}$ & $\begin{array}{l}\text { Rangos } \\
\text { X Variable }\end{array}$ \\
\hline \multirow{3}{*}{$\begin{array}{l}\text { Desarrollo de } \\
\text { capacidades } \\
\text { socioafectivas }\end{array}$} & $\begin{array}{l}\text { Dimensión } \\
\text { Interpersonal }\end{array}$ & $\begin{array}{c}1,2,3,4,5,6 \\
, 7,8\end{array}$ & 8 & $\begin{array}{l}\text { R1: } 1 \text { - Deficiente } \\
\text { R2: } 2 \text { - Suficiente } \\
\text { R3: } 3 \text { - Bueno } \\
\text { R4: } 4 \text { - Excelente }\end{array}$ & $\begin{array}{l}\mathrm{R} 1:<8-16] \\
\mathrm{R} 2:<16-21] \\
\mathrm{R} 3:<21-29] \\
\mathrm{R} 4:<29-32]\end{array}$ & \multirow{3}{*}{$\begin{array}{l}\text { MIN=18 } \\
\text { MAX=72 } \\
\text { Rango 1: }<18-36] \\
\text { Rango 2: }<36-48] \\
\text { Rango } 3:<48-66] \\
\text { Rango } 4:<66-72]\end{array}$} \\
\hline & $\begin{array}{l}\text { Dimensión } \\
\text { Intrapersonal }\end{array}$ & $\begin{array}{c}9,10,11 \\
12,13,14\end{array}$ & 6 & $\begin{array}{l}\text { R1: } 1 \text { - Deficiente } \\
\text { R2: } 2 \text { - Suficiente } \\
\text { R3: } 3 \text { - Bueno } \\
\text { R4: } 4 \text { - Excelente }\end{array}$ & $\begin{array}{l}\mathrm{R} 1:<6-12] \\
\mathrm{R} 2:<12-16] \\
\mathrm{R} 3:<16-22] \\
\mathrm{R} 4:<22-24]\end{array}$ & \\
\hline & $\begin{array}{l}\text { Dimensión de } \\
\text { Adaptabilidad }\end{array}$ & $\begin{array}{l}15,16,17,1 \\
8\end{array}$ & 4 & $\begin{array}{l}\text { R1: } 1 \text { - Deficiente } \\
\text { R2: } 2 \text { - Suficiente } \\
\text { R3: } 3 \text { - Bueno } \\
\text { R4: } 4 \text { - Excelente }\end{array}$ & $\begin{array}{l}\mathrm{R} 1:<4-08] \\
\mathrm{R} 2:<08-11] \\
\mathrm{R} 3:<11-15] \\
\mathrm{R} 4:<15-16]\end{array}$ & \\
\hline \multirow{4}{*}{$\begin{array}{l}\text { Comportamien } \\
\text { to Pro } \\
\text { Ambiental }\end{array}$} & $\begin{array}{l}\text { Acciones } \\
\text { orientadas al } \\
\text { Medio Ambiente }\end{array}$ & $\begin{array}{c}1,2,3,4,5,6 \\
, 7,8,9\end{array}$ & 9 & $\begin{array}{l}\text { R1: } 1 \text { - Deficiente } \\
\text { R2: } 2 \text { - Suficiente } \\
\text { R3: } 3 \text { - Bueno } \\
\text { R4: } 4 \text { - Excelente }\end{array}$ & $\begin{array}{l}\mathrm{R} 1:<9-18] \\
\mathrm{R} 2:<18-24] \\
\mathrm{R} 3:<24-33] \\
\mathrm{R} 4:<33-36]\end{array}$ & \multirow{4}{*}{$\begin{array}{l}\text { MIN=16 } \\
\text { MAX=64 } \\
\text { Rango 1: }<16-32 \\
\text { Rango 2: }<32-43] \\
\text { Rango } 3:<43-59] \\
\text { Rango } 4:<59-64]\end{array}$} \\
\hline & $\begin{array}{l}\text { Acciones } \\
\text { orientadas a la } \\
\text { Educación de la } \\
\text { Salud }\end{array}$ & $\begin{array}{c}10,11,12,1 \\
3,14\end{array}$ & 5 & $\begin{array}{l}\text { R1: } 1 \text { - Deficiente } \\
\text { R2: } 2 \text { - Suficiente } \\
\text { R3: } 3 \text { - Bueno } \\
\text { R4: } 4 \text { - Excelente }\end{array}$ & $\begin{array}{l}\mathrm{R} 1:<5-10] \\
\mathrm{R} 2:<10-13] \\
\mathrm{R} 3:<13-18] \\
\mathrm{R} 4:<18-20]\end{array}$ & \\
\hline & $\begin{array}{l}\text { Acciones } \\
\text { orientadas a la } \\
\text { Prevención de } \\
\text { situaciones de } \\
\text { Riesgo }\end{array}$ & 15,16 & 2 & $\begin{array}{l}\text { R1: } 1 \text { - Deficiente } \\
\text { R2: } 2 \text { - Suficiente } \\
\text { R3: } 3 \text { - Bueno } \\
\text { R4: } 4 \text { - Excelente }\end{array}$ & $\begin{array}{l}\mathrm{R} 1:<2-04] \\
\mathrm{R} 2:<04-05] \\
\mathrm{R} 3:<05-07] \\
\mathrm{R} 4:<07-08]\end{array}$ & \\
\hline & & TOTAL & 34 & & & \\
\hline
\end{tabular}

La ficha de observación a niños de inicial se estructuró considerando las dos variables de estudio: capacidades socio afectivas del niño observado y sus dimensiones y comportamientos proambientales del niño observado y sus dimensiones.

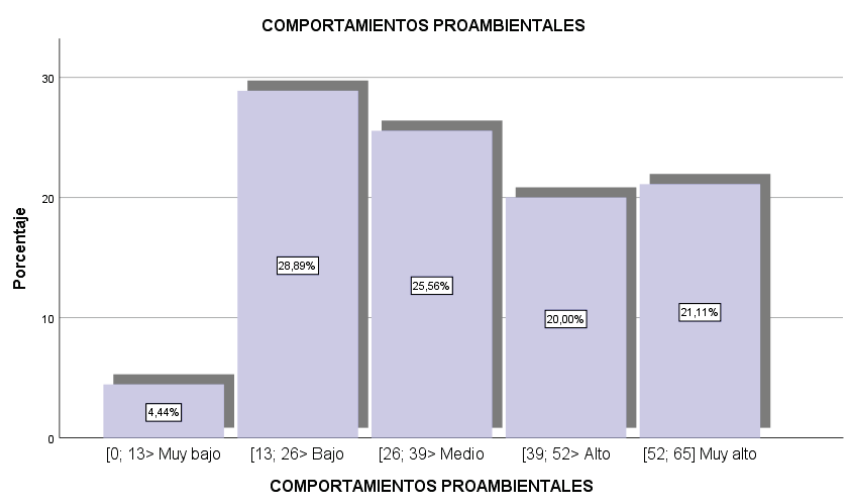

\section{RESULTADOS}

Análisis de las variables Capacidades socioafectivas y comportamientos proambiental

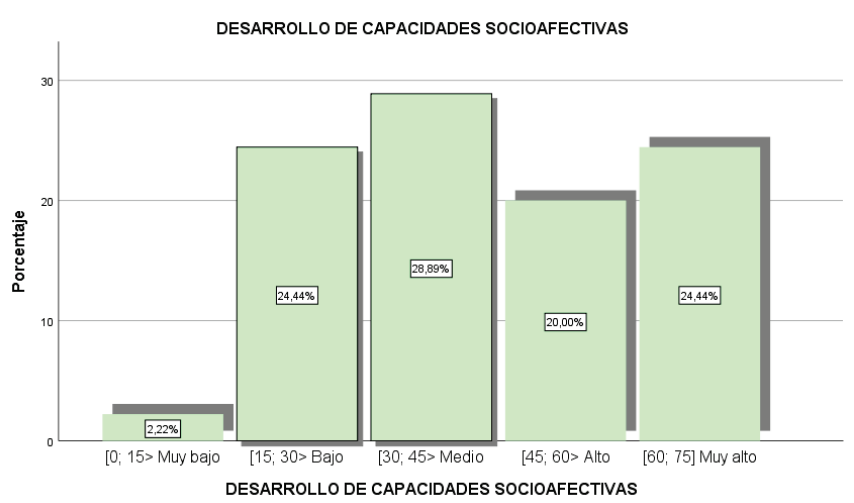

Figura 1. Histograma dispersión entre desarrollo de capacidades socioafectivas y comportamientos proambientales

Se observa que las variables Comportamientos proambientales tiene medidas de tendencia central a rededor de 35 puntos de un total de 64; también se observa que la mayor cantidad de la muestra se ubica en el intervalo [13; 26> con un porcentaje de $28,86 \%$ que ha sido nombrado como bajo; como las medidas de tendencia central no se encuentran en dicho intervalo se puede calificar que la distribución correspondiente, de manera general, a una calificación como baja. Existe una mayor cantidad de participantes evaluados a la derecha por lo cual la mediana está orientada a la izquierda de la media. Se puede observar también que las demás categorías tienen valores significativos; Muy alto $21,11 \%$, Alto $20 \%$, medio 25,56 mostrando que respecto de los Comportamientos proambientales los estudiantes tienen diferentes formas de mostrar esta característica.

Se aprecia que las variables Desarrollo de capacidades socioafectivas tiene medidas de tendencia central a rededor de 42 puntos de un total de 72; también se observa que la mayor cantidad de la muestra se ubica en el intervalo [30; 45> con un porcentaje de $28,89 \%$ que ha sido nombrado como bajo; como las medidas de 
tendencia central se encuentran en dicho intervalo se puede calificar que la distribución correspondiente de manera general a una calificación como media. Existe una mayor cantidad de participantes evaluados a la derecha por lo cual la mediana está orientada a la izquierda de la media. Se puede observar también que las demás categorías tienen valores significativos; Muy alto $24,44 \%$, Alto $20,02 \%$, Bajo $24,44 \%$ mostrando que respecto de los Desarrollo de capacidades socioafectivas los estudiantes tienen diferentes formas de mostrar esta característica.

Tabla 4.

Estadísticos de desarrollo de capacidades socioafectivas y comportamientos proambientales

\begin{tabular}{|c|c|c|c|}
\hline \multicolumn{4}{|c|}{ Estadísticos } \\
\hline & & $\begin{array}{c}\text { Desarrollo de } \\
\text { capacidades } \\
\text { socioafectivas }\end{array}$ & $\begin{array}{c}\text { Comportamientos } \\
\text { proambientales }\end{array}$ \\
\hline \multirow[t]{2}{*}{$\mathrm{N}$} & Válido & 90 & 90 \\
\hline & Perdidos & 0 & 0 \\
\hline Media & & 42,66 & 35,51 \\
\hline Mediana & & 41,50 & 33,00 \\
\hline Moda & & 50 & 0 \\
\hline $\begin{array}{l}\text { Desv. } \\
\text { Desviación }\end{array}$ & & 17,819 & 16,070 \\
\hline $\begin{array}{l}\text { Coeficiente } \\
\text { de variación }\end{array}$ & & $41,780 \%$ & $45,250 \%$ \\
\hline
\end{tabular}

El coeficiente de variación de las distribuciones muestra que las variables tienen un nivel de desarrollo muy parecido $(41,78<45,25)$ se puede decir que la distribución de la Desarrollo de capacidades socioafectivas muestra un desarrollo más homogéneo que la distribución de la variable Comportamientos proambientales; sin embargo, esta diferencia es poco relevante, por lo que se puede decir que los variables tiene un desarrollo parecido en sí (Tabla 4).

Para estimar la correlación estadística entre dos variables medidas con un nivel ordinal, se utiliza el coeficiente de correlación de Spearman, del análisis no paramétrico. En este sentido, no se requiere de presupuestos acerca de la forma de distribución de la población.

\section{Tabla 5.}

Relación entre el desarrollo de capacidades socioafectivas y comportamientos proambientales

\begin{tabular}{|c|c|c|c|}
\hline \multicolumn{2}{|c|}{ Correlación } & & \multirow{2}{*}{$\begin{array}{l}\text { Comportamie } \\
\text { ntos } \\
\text { proambientale } \\
\mathrm{s} \\
\qquad 0,976\end{array}$} \\
\hline \multirow{3}{*}{$\begin{array}{l}\text { Rho de } \\
\text { Spearman }\end{array}$} & \multirow{3}{*}{$\begin{array}{l}\text { Desarrollo de } \\
\text { capacidades } \\
\text { socioafectivas }\end{array}$} & Correlación & \\
\hline & & $\begin{array}{l}\text { Sig. } \\
\text { (bilateral) }\end{array}$ & 0,000 \\
\hline & & $\mathrm{N}$ & 90 \\
\hline
\end{tabular}

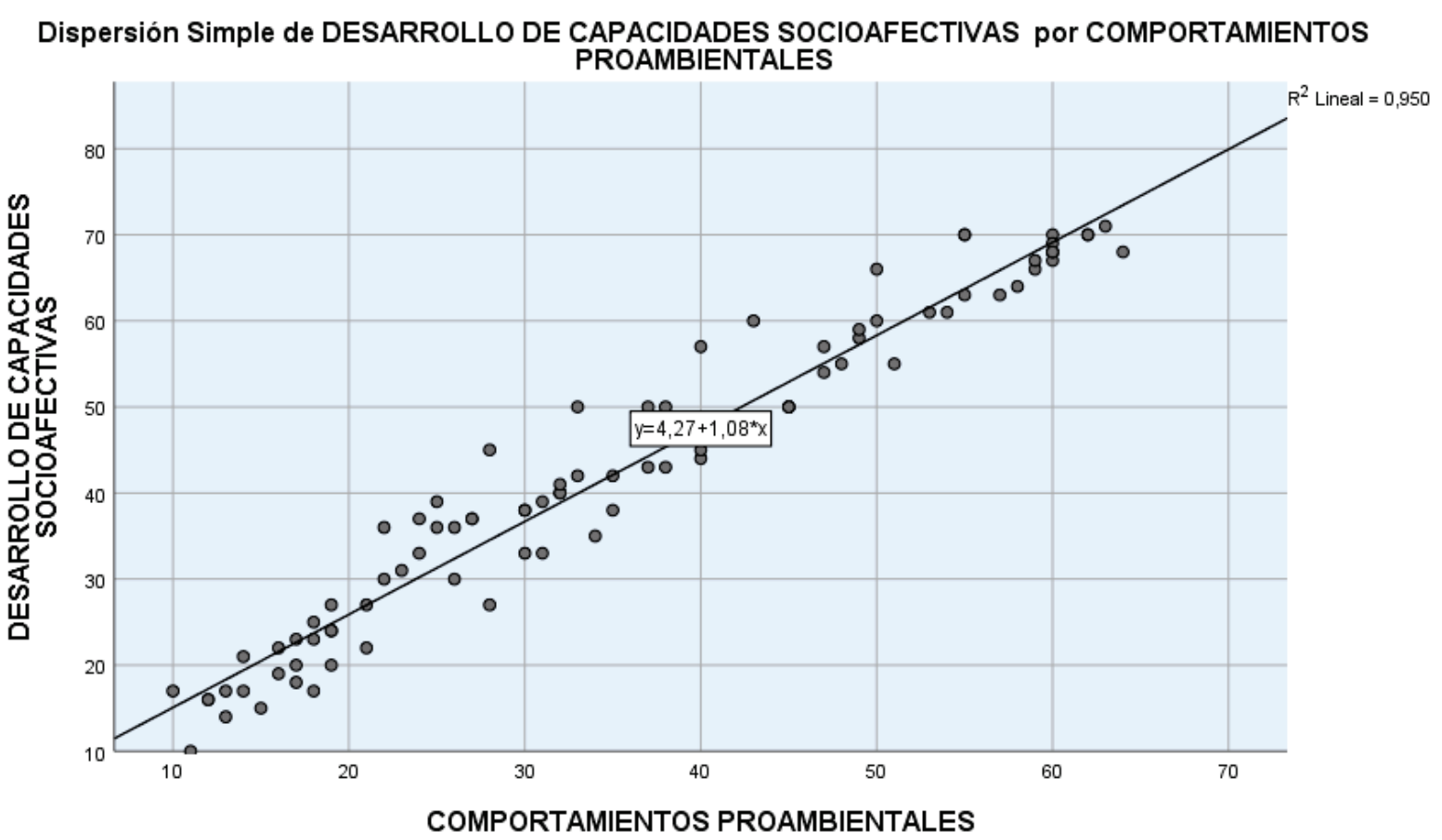

Figura 2. Gráfico de dispersión entre desarrollo de capacidades socioafectivas y comportamientos proambientales 
De acuerdo con los resultados de la correlación, nos muestran que $p=0,00$ como coeficiente de significancia y un coeficiente Rho de Spearman $(\mathrm{Ro}=0,976)$ se puede decir que se acepta el hecho que existe una relación directa y significativamente positiva entre el Desarrollo de capacidades socioafectivas y Comportamientos proambientales Tabla 5). Esta relación es lineal y dependiendo Desarrollo de capacidades socioafectivas $Y=1,08^{*} X+4,27$ donde $X$ es la variable Comportamientos proambientales; es necesario mencionar que la existencia de esta dependencia no implica necesariamente una dependencia causa efecto entre las variables (Figura 2).

\section{DISCUSIÓN}

Los resultados obtenidos en lo que concierne a comportamientos proambientales no coincide con los reportados por Vera (2017) quien halló que un $94 \%$ de la población evaluada se mantenían en el nivel de actividades medio, en relación al resultado de $28,86 \%$, encontrándose una diferencias muy fuertes, esto debido a que el grupo de estudio fueron diferentes, adolescentes en el caso de Vera (2017) y en niños en esta investigación.

Sin embargo estos resultados coincide con lo reportado por Fuentealba (2018) quién encontró un nivel muy bajo de la valoración actitudinal proambiental; esta valoración lo realizaron se hizo mediante un estudio cualitativo. En este caso el estudio muestra que las estrategias sociales no permitieron el desarrollo de las actitudes proambientales corroborando lo encontrado en el trabajo y lo mostrado en la presente investigación; donde el nivel de las actividades proambientales también fue bajo. Es importante recalcar que Fuentealba no realizó un análisis correlacional, por tanto, no es posible comparar a este nivel, pero coinciden en el nivel de las actividades de proambientales, empero, difieren en el tipo de investigación.

Por otro lado, Carrasco (2016), encontró que la mayoría de los estudiantes evaluados presentaron un nivel bajo en la actitud hacia la conservación del ambiente con un $28 \%$ lo cual corrobora lo encontrado en este trabajo de investigación $(28,86 \%)$, donde el nivel también es bajo. Además, realizó la correlación de la inteligencia emocional con las actitudes de conservación al medio ambiente mostrando, en este caso, encontró que existe un nivel de correlación alto con un coeficiente de Pearson de 0,859 , que es semejante al encontrado en el trabajo expuesto $(0,979)$. Por tanto, se puede afirmar que existe una convergencia en el nivel de actitudes proambientales.

\section{CONCLUSIONES}

El Desarrollo de las Capacidades Socioafectivas tiene una relación con el Comportamiento Proambiental de los niños de la Institución Educativa Inicial $N^{\circ} 068$ del distrito ElAgustino de la ciudad de Lima.

La relación entre las variables; Desarrollo de las
Capacidades Socioafectivas con el Comportamiento Proambiental es lineal mostrando un ajuste $95 \%$ a la recta de aproximación $Y=1,08^{*} X+4,27$.

La variable comportamientos proambientales tienen un nivel de desarrollo en promedio bajo con porcentaje del $28,86 \%$ en esta categoría.

La variable Desarrollo de capacidades socioafectivas tienen un nivel de desarrollo en promedio medio con porcentaje del $28,86 \%$ en esta categoría.

\section{REFERENCIAS}

Carrasco, M. G. (2016). La inteligencia emocional y las actitudes para la conservacion del medio ambiente en los alumnos de educación secundaria de las i.e. públicas del distrito de Chucuito, Tesis de Magister. Universidad Nacional San Agustín.

Duránd, M., Alzate, M., López, W., \& Sabucedo, J. M. (2007). Emociones y comportamiento pro ambiental. Revista Latinoamérica de Psicología, 39(2), 287-296.

Fuentealba, M. (2018). Valoración actitudinal proambiental: un análisis global en estudiantes de enseñanza primaria, secundaria y terciaria. Luna Azul, 159-177. doi:10.17151/luaz.2019.47.9

Kollmuss, A., \& Agyeman, J. (2002). Tenga en cuenta la brecha: ¿por qué las personas actúan ambientalmente y cuáles son las barreras para el comportamiento proambiental? Revista de investigación en educación ambiental, 8(3), 239-260.

Martínez, J. (2004). Comportamiento proambiental. Una aproximación al estudio del desarrollo sustentable con énfasis en el comportamiento persona-ambiente. Theomai(99).

Papalia, D., Wendkos, S., \& Feldman, R. D. (2007). Desarrollo humano. Washington D.F.: McGraw-Hill.

Peña, D. G. (2017). Creencias y Comportamientos Proambientales en Estudiantes de Administración en Universidades mexicanas en función del grado de implementación del Sistema de Gestión Ambiental (SGA) . Barcelona.

Vera, A. M. (2017). Actitudes Proambientales en adolescentes de la Asociación Educación, Protagonismo y Arte del Asentamiento Humano San Cosme - La Victoria; 2016, Tesis doctoral. Universitat de Barcelona.

Zapata, R. L., \& Castrechini, Á. (2011). Conducta proambiental y personalidad: Análisis de un barrio de Lima. Quaderns de psicología, 13(1), 47-61. 